\title{
INTRODUCTION
}

\section{Reproductive decision-making: towards improved theoretical, methodological and empirical approaches}

\author{
Maria Rita Testa, Tomáš Sobotka and S. Philip Morgan*
}

This issue of the Vienna Yearbook of Population Research features contributions presented at the conference "From intentions to behaviour: reproductive decisionmaking in a macro-micro perspective" organised by the Vienna Institute of Demography of the Austrian Academy of Sciences and held in Vienna in December 2010. The meeting included a presentation of the findings of the multidisciplinary research project "REPRO" (Reproductive decision making in a macro-micro approach) on the antecedents and outcomes of fertility intentions. One of the contributions of the project was to show that the stylised 'gap' between intended and actual fertility behaviour - which suggests plenty of scope for policy action - provides crude and potentially misleading simplifications of the complex picture of reproductive decision-making and, consequently, of the role of family-related policies in Europe (Testa and Philipov 2011). An important part of this complexity emerges from the interaction between individual and aggregatelevel factors which determine fertility decisions and the related birth outcomes. In a macro-micro perspective, fertility rates depend on the conditions prevailing in a society. These 'macro' conditions do not directly affect fertility but rather impinge on the fertility decision-making processes of individuals and couples. Hence, a society's fertility rates are the aggregate-level result of the myriad of fertility decisions taken by individuals and couples. The main challenge of the REPRO project was to investigate fertility decision-making in an integrated macro-micro framework by looking at each of the five different linkages schematically represented in Figure 1.

* Maria Rita Testa (correspondence author), Vienna Institute of Demography, Austrian Academy of Sciences, Wittgenstein Centre for Demography and Global Human Capital, Wohllebengasse 12-14, 6th floor, 1040 Vienna, Austria. Email: maria.rita.testa@oeaw.ac.at

Tomáś Sobotka, Vienna Institute of Demography, Austrian Academy of Sciences, Wittgenstein Centre for Demography and Global Human Capital, Vienna, Austria.

Philip S. Morgan, Sociology Department and Social Science Research Institute, Duke University, Durham, North Carolina, USA. 
Figure 1:

A micro-macro model of fertility

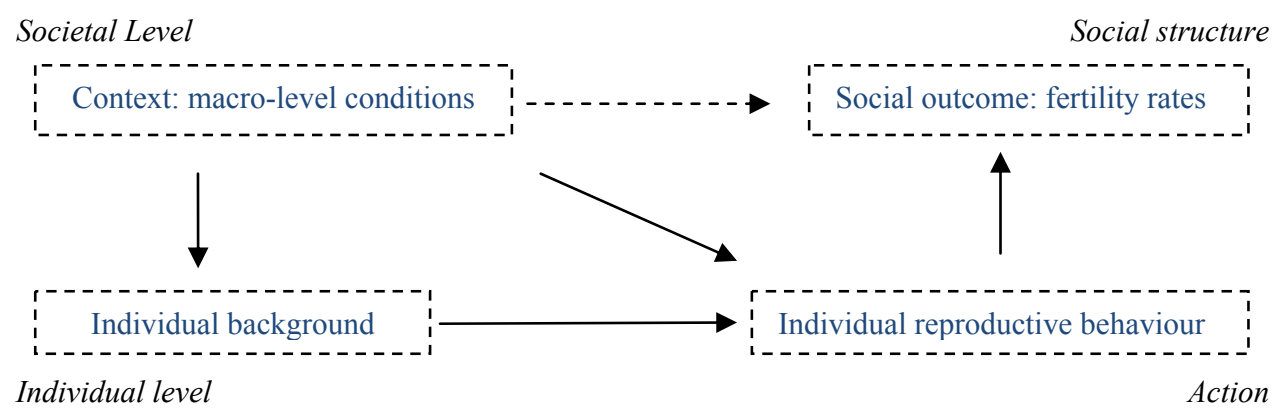

Source: inspired by Coleman 1990

In particular, the REPRO scholars demonstrated that the societal, economic and cultural environment play a role not only in the macro-level relationships (the horizontal arrow in Figure 1) but also in a macro-micro link (the diagonal arrow in Figure 1). For instance, the societal norm about the disapproval of a mother with a child below age three getting a full-time job exerts a negative impact on the individual's prospective childbearing preferences even after controlling for the individual background characteristics. The key findings suggest that beyond crucial factors related to partnership, education, employment and housing conditions as well as to work-life balance, fertility decisions are influenced by the normative pressure of relatives and friends as well as by personal attitudes towards having a child which are especially important when the decision concerns the first child. These two latter factors together with the ability to perform the behaviour are the main determinants of intentions in the Theory of Planned Behaviour (TPB) proposed by Ajzen (1985; Fishbein and Ajzen 2010). One of the important advances in the scientific understanding of reproductive intentions stems from the REPRO project's concerns with the first systematic application of the TPB to fertility research. New longitudinal data in a growing number of European countries, such as the Generations and Gender Surveys, include a set of questions specifically designed for an operationalisation of the TPB. Thus, the REPRO project seized the unique opportunity to test the TPB and to assess its value for fertility and, more generally, for demographic research.

\section{The debate on the Theory of Planned Behaviour and its usefulness in understanding reproductive decisions}

The TPB takes a most prominent role in this volume of the Vienna Yearbook of Population Research. Many contributions (7 out of 12, if we exclude the debate section) make explicit reference to the TPB. Moreover, the debate section is developed and organised around the appropriateness and utility of this theoretical framework for the study of reproductive decision-making. The TPB has greatly 
enhanced our understanding of the development process of individuals' fertility intentions by providing a useful theoretical tool for the study of fertility decisionmaking. Its application to the specific case of human reproductive behaviour, however, requires a thoughtful reflection about its strengths and limits as well as a discussion about the directions along which it might be improved. To this end eight scholars have contributed their reflections on the TPB merits which are presented in the debate section of this volume. The debate has been stimulated by an opening contribution by Morgan and Bachrach, who identify four main aspects of the TPB that should be improved. Besides 1) the postulation of a clear intent preceding the reproductive behaviour, they see a need for an explicit consideration of: 2) the interdependent nature of fertility outcomes which require a series of decisions interacting with other life behaviours, 3) the developmental nature of intentions that may change over individuals' life course, and 4) the role of macro social context in which individuals and their intentions are formed. As outlined by the other contributors of the debate, with adequate data, the TPB can incorporate a dynamic life-course trajectories approach (Barber, Klobas, Liefbroer, Philipov) and can be adjusted to address the complexity of fertility as a product of multiple behaviours (Barber, Philipov); moreover, it is flexible enough to allow for creative thinking about links between societal and individual-level factors (Klobas, Liefbroer, Philipov). However, as underlined by all the authors of the debate, having a child is not always the outcome of reasoned action, and unintended childbearing may be driven by motivations that do not reflect conscious decisions to pursue a given target (Barber, Miller).

During the VID conference, Ajzen (2010) clarified that the close link between intentions and subsequent behaviour holds true only if the behaviour is specified in all of its four components - the target, the action, the context and the time - and that the partner is of crucial importance to specify the context. The TBP, however, does not explicitly consider the dyadic nature of reproduction (Miller, Philipov). Moreover, while the perception of a disagreement with the partner influences an individual's normative beliefs and, consequently, his/her fertility intentions, the motivation to comply with the partner does not adequately describe the influence and disagreement effects of couple decisional conflicts (Miller). Hence, demographers should do additional work to make the TPB more appropriate for fertility research.

On the one hand, the fact that many demographers have used the TPB in their fertility analyses signals its usefulness for the analysis of the determinants of reproduction and reproductive decision-making. On the other hand, it is legitimate to try and overcome the difficulties related to the implementation of the TPB for the case of fertility. Of course the TPB should be adjusted to the peculiarities of the childbearing process but competing alternative theories should also be considered. For instance, the search for alternative theoretical approaches outlined in this volume (Morgan and Bachrach, debate section, and an article by Ní 
Bhrolcháin and Beaujouan), could offer fruitful ways to move beyond the existing state of the art in fertility research.

The debate section calls for greater clarity of both the construct and the measurement of intention (Philipov) and for a critical look at the central role of intentions in fertility research (Miller). Alternative theories that seek to explain fertility not only as an individual function of environmental opportunities and constraints but also as a direct function of individuals' perceptions or interpretive frameworks should be considered in future research (Bachrach and Morgan).

\section{The contributions to the Yearbook}

The first four research articles in this volume propose methodological approaches to the study of fertility preferences which may refine the existing measurement of childbearing intentions and make the match between birth desires or intentions and reproductive behaviour more accurate.

In Differences between fertility desires and intentions: implications for theory, research and policy, Warren Miller compares the two constructs of intentions and desires as conceptualised in his three-step motivational sequence of fertility decision-making which starts with motivational traits, continues with fertility desires and concludes with fertility intentions. He suggests that desires, which are the relevant determinants of intentions, may sometimes 'bypass' the latter and act directly on behaviour to influence fertility outcomes. He highlights an important difference between the two constructs. Unlike with intentions, it is psychologically possible for individuals of reproductive age to simultaneously desire and not desire a(nother) child. For this reason it would be preferable to measure both dimensions of childbearing desires, positive and negative, on separate unipolar scales. This innovation would enable investigators to study the interaction between the positive and negative valences of desires and to address the phenomenon of ambivalence (given by high positive and high negative desires) and indifference (given by low positive and low negative desires) helping to generate a new understanding of unintended pregnancy.

In the article Uncertainty in fertility intentions in Britain, 1979-2007, Máire Ní Bhrolcháin and Éva Beaujouan use time series of repeated rounds of the General Household Survey in Great Britain to study uncertainty in fertility intentions. They show a relatively high prevalence of uncertainty about prospective childbearing in Britain in the range between $10 \%$ and $40 \%$, which is consistent with the evidence presented for other developed countries. They demonstrate that by providing explicit precodes for uncertainty in an otherwise unchanging survey context triples the reporting of uncertain fertility intentions from $9 \%$ in 1990 to $26 \%$ in 1991 . The authors question the usefulness of traditional approaches to measuring fertility intentions and call for quantitative and qualitative research to develop new concepts and to validate new measures of fertility expectations together with indicators of certainty and uncertainty. Uncertainty is seen as a rational response to the evolving life course which might 
reflect an individual's different states such as ambivalence and indecision. Therefore, a new theoretical approach should incorporate uncertainty in the family formation process.

Saskia Hin and colleagues provide another perspective on uncertainty. In Fertility preferences: what measuring second choices teaches us, they study fertility ideals considering a hierarchy of reproductive preferences rather than a single target. This methodology has been tested in an online survey carried out in the Netherlands in 2010 that included, in addition to the respondents' first preference, also their second, third, and fourth alternative choice of the ideal number of children. The findings reveal that the psychological distance between specified numbers of children is not the same for all individuals. The Dutch respondents are consistently more open to an alternative that entails lowering their fertility ideal than to a choice that surpasses their first ideal number of offspring. The authors argue that by capturing a range of possibilities an individual may choose from one can better understand the effect of broader social norms on the reported first choice, which may reflect a socially desirable answer instead of the personal family size ideal. Moreover, the variance between social groups can be better measured because second and further choices amplify differences between groups. As the authors stress, the degree of individuals' willingness to substitute one ideal for another under different circumstances gives useful insights on why many people in low-fertility societies may deviate in their behaviour from their initially stated ideal.

Couple disagreement counts among the most pertinent reasons why some individuals do not fulfil their reproductive intentions or ideals. In the contribution Couples' childbearing behaviour in Italy: which of the partners is leading it?, Maria Rita Testa, Laura Cavalli and Alessandro Rosina use longitudinal data for Italian couples (2003-2007) to examine the influence of each of the partners on childbearing decision-making in case of couple conflict. They show that at the beginning of the family formation process women have more power in decisionmaking while after the birth of the first child a disagreement between partners produces the same inhibiting effects on reproduction irrespective of whether the man wants to have a child while the women does not or vice versa. The authors demonstrate that models including both partners' fertility intentions predict reproductive behaviour more accurately than models based on only one partner's intentions. They call therefore for collection of data from both partners.

Testa et al. provide a transition to the next three articles that focus on the relationship between reproductive intention and subsequent behaviour. The first two studies aim to investigate the role of family networks and, more generally, of social learning and social influence. The third one addresses the relevance of the individual's socio-economic status in the formation and implementation of reproductive plans.

In The influence of the family network on the realisation of fertility intentions, Nicoletta Balbo and Melinda Mills use two waves of Netherlands Kinship Panel 
Survey to investigate the family network's influence on the realisation of shortterm fertility intentions. Their findings show that strong family social capital, as measured by the strength of family ties and family networks, is associated with a lower probability to realise the intention to have a second or higher-order child. Thus, being strongly embedded in one's family of origin might discourage from realising the intention to have another child, as "people who already have very satisfying family ties and a strong family network lack the motivation to enact their positive fertility intentions". The authors also show that having a sibling with a young child below age 12 is positively associated with the realisation of the short-term fertility intentions. They call for additional research that makes use of more extensive social network data to gain further insights into the process of social influence and social learning.

In their contribution Intergenerational transmission of fertility intentions and behaviour: the role of contagion, Markus Kotte and Volker Ludwig use the first and the second wave of the "German Panel Analysis of Intimate Relationships and Family Dynamics" (Pairfam) to investigate the intergenerational transmission of fertility intentions and behaviour. They focus on two main mechanisms: transmission from the family of origin, as measured by the number of siblings, and 'contagion' by their siblings' fertility behaviour. They find a strong influence of the number of siblings for both intentions and behaviour. However, the effect of 'contagion' by siblings with young children is weak for family size preferences and almost non-existent for actual fertility behaviour. By contrast, the authors report a significant peer influence, suggesting that friends and significant others who recently had a child have a positive influence on short-term fertility preferences as well as on actual fertility. The availability of additional waves of the Pairfam study will allow the authors to perform more in-depth analysis and to overcome the selectivity and endogeneity problems inherent in their data.

Socioeconomic status plays an important role in fertility intentions and the behaviour of women in Flanders. David de Wachter and Karel Neels show in Educational differentials in fertility intentions and outcomes: family formation in Flanders in the early 1990s, that a tertiary level of education was linked to both higher lifetime and short-term (within three years) fertility intentions than lower education degrees in 1991. Moreover, detailed fertility histories, extracted from the population census of 2000, reveal that women with high-level education also had the highest intensity of childbearing during economically uncertain times in 1992-94. Full-time employment facilitated family formation, indicating that access to stable employment and childcare availability have become important preconditions for the realisation of first-birth intentions, especially among tertiary-educated. Also the progression to the second and third birth was more frequent among more highly educated women. Among mothers, part-time employment had a positive effect on the progression to a second and particularly a third birth; however, less educated women experienced a weakening of their 
economic position after first birth as a result of high unemployment and inactivity.

A comparative perspective is extremely fruitful for the explanation of fertility desires and fertility-related behaviours, when institutional and historical context is taken into account. In Family policies, norms about gender roles and fertility decisions in France and Germany, Clémentine Rossier, Sara Brachet and Anne Salles use qualitative data to compare fertility decisions in France and western Germany, two neighbouring countries with different fertility levels and attitudes as well as different family-related policies, gender role attitudes and child care norms. Rossier et al. suggest that social norms about the compatibility of motherhood and work outside the family have a crucial importance in explaining the differences between the two countries. Although women in France as well as Germany have rather similar visions of gender roles, they show strong awareness of what the authors perceive as the nationally dominant models of child care. These dominant norms of the prominence of mother-provided child care in western Germany and of a shared child care system (mother and child care institutions) in France strongly influence individual fertility decisions. In particular the authors argue that in western Germany women who want or need to work may decide not to have a(nother) child because they are not ready to use external child care services even if they are available and affordable. In France, in contrast, the acceptance of external child care allows women who work and decide to have children to create and rely on external child care solutions even when the institutional supply is scarce.

Anna Rotkirch, Stuart Basten, Heini Väisänen and Markus Jokela turn their attention to men's motivation for children. In Baby longing and men's reproductive motivation, they analyse data from three recent (2007-2009) surveys conducted in Finland and combine them with excerpts from in-depth interviews of parents with three or more children. Defining baby longing (or 'baby fever') as "a visceral emotional and physical desire to have a baby" they report that this is not an exclusively female emotion: nearly one-half (44\%) of men aged 20-59 had longed for a baby at least once in their life. Most commonly, baby longing occurs to men around the time of deciding for their first child, thus presumably interacting with their fertility desires and plans, but it also often emerges while trying to achieve pregnancy. It is closely related to union formation, which may serve as a trigger of the 'baby fever', but it is unrelated to socioeconomic status as measured by income, occupation and education level.

The last of the refereed articles is not focused on the determinants of (intended) reproductive behaviour but rather on its consequences on individuals' well-being. This impact, of course, has important effects on the decision to have an additional child. Anna Baranowska and Anna Matysiak investigate in their contribution Does parenthood increase happiness? Evidence for Poland the effects of childbearing on happiness. Panel data analysis enabled them to control for endogeneity of subjective well-being and parenthood. Their findings reveal a 
positive effect of the first child on the subjective well-being of mothers and a similar, albeit smaller and only temporary, effect for fathers. This positive effect of parenthood on happiness is not observed in the subsequent transitions to second- and higher-order births, suggesting that either the first child satisfies all the emotional needs of the parents or that the costs of rearing children increase more steeply with parity than the relative (emotional) benefits.

The Data and trends section in this volume contains two contributions describing relatively unique datasets on fertility ideals and intentions. In their article Design and implementation of an online weekly survey to study unintended pregnancies, Jennifer S. Barber, Yasamin Kusunoki and Heather Gatny describe the "Relationship Dynamics and Social Life" (RDSL) study which collects weekly journal-based attitudinal and behavioural measures of pregnancy, relationship and contraceptive use among women in early adult years aged 18-22. Rates of unintended pregnancy in the US peak at these ages and, in parallel, there is a high degree of instability in relationship histories and contraceptive use. The survey aims to address critical limitations in the existing measures of unintended pregnancy. Preliminary results show that young women experience frequent changes in their attitudes, behaviours and pregnancy desires, which all have important implications for unintended pregnancy.

In the article Couple disagreement about fertility preferences and familyfriendly policy measures in the Czech Republic, Beatrice Chromková Manea and Petr Fučík describe the findings of an ad-hoc survey "Marriage, Work and Family" conducted in the Czech Republic in 2005. The authors show that the high share of couple agreement about child-number intentions observed at the time of the survey, $80 \%$, is the final outcome of a preliminary negotiation process between the partners in most of the cases. Indeed, a higher degree of couple disagreement is registered when partners are asked to indicate their family size ideals and childbearing attitudes or to report their opinion about the potential effect of the implementation of different family-friendly policy measures on their own reproductive behaviour.

\section{Conclusion}

The contributions published in this volume focus on human reproductive decision-making from many different angles, applying individual and couple perspectives, looking both at the male and female side, taking into account the family of origin and the wider social network, applying vastly different time scales (from weekly measures up to lifetime indicators of intentions and behaviour), and using different data (ranging from qualitative interviews to quantitative analyses and from cross-sectional to time series and longitudinal studies). Also the range of underlying factors and mechanisms studied is wide, including difficult-to-conceptualise aggregate notions of national child care norms, individual emotions pertaining to reproductive motivation (baby longing) 
or the emotional benefits thereof (happiness). The variety of methods and approaches used attests to the fact that human reproductive behaviour is extremely complex. At the heart of the REPRO project and many other studies presented here was the need to profoundly examine the causes and the consequences of the mismatch between fertility intentions and behaviour. This recurring theme stimulates and challenges demographers for improvements in theoretical, methodological and analytical approaches. We hope that this special issue on reproductive decision-making - which has gone far beyond the results achieved in the REPRO project - will encourage additional critical and creative thinking about existing theoretical, methodological and empirical frameworks as well as further steps toward the creation of alternative, perhaps more general and useful, models of human fertility and reproduction.

\section{References}

Ajzen, I. 1985. From intentions to actions: a theory of planned behavior. In Action control: from cognition to behaviour, ed. J. Kuhl and J. Beckmann, 11-39. Berlin: Springer.

Ajzen, I. 2010. Fertility intentions and the Theory of Planned Behaviour. Paper presented at the Conference: "From intentions to Behaviour: Reproductive Decision Making in a Macro-Micro Perspective", Vienna, 2-3 December 2010. http://www.oeaw.ac.at/vid/in2b/

Coleman, J.S. 1990. Foundations of social theory. Cambridge: The Belknap Press of Harvard University Press.

Fishbein, M. and I. Ajzen 2010. Predicting and changing behavior: the reasoned action approach. New York: Psychology Press.

Testa, M.R. and D. Philipov 2011. Few children in Europe: a deliberate choice or a compromise? European Policy Brief. http://www.oeaw.ac.at/vid/repro 
